\title{
(2) OPEN ACCESS \\ Swept-source OCTA quantification of capillary closure predicts ETDRS severity staging of NPDR
}

\author{
Torcato Santos, ${ }^{1}$ Lewis H Warren, ${ }^{2}$ Ana Rita Santos (10 , 1,3 Inês Pereira Marques, 1,4 \\ Sophie Kubach, ${ }^{2}$ Luís G Mendes, ${ }^{1}$ Luis de Sisternes, ${ }^{2}$ Maria H Madeira, 1,4 \\ Mary Durbin, ${ }^{2}$ Jose G Cunha-Vaz (D) 1,4
}

\begin{abstract}
${ }^{1} A|B| L I$ - Association for Innovation and Biomedical Research on Light and Image, Coimbra, Portugal

${ }^{2}$ R\&D, Carl Zeiss Meditec, Dublin, California, USA ${ }^{3}$ Department of Orthoptics, School of Health, Polytechnic of Porto, Porto, Portugal

${ }^{4}$ Coimbra Institute for Clinical and Biomedical Research (iCBR), Faculty of Medicine, University of Coimbra, Coimbra, Portugal
\end{abstract}

\section{Correspondence to}

Professor Jose G Cunha-Vaz, AlBILI, Coimbra, Coimbra, Portugal; cunhavaz@aibili.pt

Received 2 September 2020 Revised 20 October 2020 Accepted 5 December 2020

\section{Check for updates}

(c) Author(s) (or their employer(s)) 2020. Re-use permitted under CC BY-NC. No commercial re-use. See rights and permissions. Published by BMJ.

To cite: Santos T, Warren $L H$, Santos AR, et al.

Br J Ophthalmol Epub ahead of print: [please include Day Month Year]. doi:10.1136/ bjophthalmol-2020-317890

\section{ABSTRACT}

Purpose To test whether a single or composite set of parameters evaluated with optical coherence tomography angiography (OCTA), representing retinal capillary closure, can predict non-proliferative diabetic retinopathy (NPDR) staging according to the gold standard ETDRS grading scheme.

Methods 105 patients with diabetes, either without retinopathy or with different degrees of retinopathy (NPDR up to ETDRS grade 53), were prospectively evaluated using swept-source OCTA (SS-OCTA, PlexElite, Carl Zeiss Meditec) with $15 \times 9 \mathrm{~mm}$ and $3 \times 3 \mathrm{~mm}$ angiography protocols. Seven-field photographs of the fundus were obtained for ETDRS staging. Eyes from agematched healthy subjects were also imaged as control.

Results In eyes of patients with type 2 diabetes without retinopathy or ETDRS levels 20 and 35, retinal capillary closure was in the macular area, with predominant alterations in the parafoveal retinal circulation (inner ring). Retinal capillary closure in ETDRS stages 43-53 becomes predominant in the retinal midperiphery with vessel density average values of $25.2 \pm 7.9(p=0.001)$ in ETDRS 43 and $23.5 \pm 3.4(p=0.001)$ in ETDRS 47-53, when evaluating extended areas of $15 \times 9$ protocol. Combination of acquisition protocols $3 \times 3 \mathrm{~mm}$ and $15 \times 9 \mathrm{~mm}$, using SS-OCTA, allows discrimination between eyes with mild NPDR (ETDRS 10, 20,35) and eyes with moderate-to-severe NPDR (ETDRS grades 43-53).

Conclusions Retinal capillary closure, quantified by SS-OCTA, can identify NPDR severity progression. It is located mainly in the perifoveal retinal capillary circulation in the initial stages of NPDR, whereas the retinal midperiphery is predominantly affected in moderate-to-severe NPDR.

\section{INTRODUCTION}

Diabetic retinopathy (DR) is one of the leading causes of blindness worldwide and the prevalence of vision-threatening DR is expected to double in the next decade. ${ }^{12}$ Considering that more than $90 \%$ of the cause of vision loss can be prevented, ${ }^{3}$ accurate staging and classification of DR are fundamental to guide treatment decisions and identify progression.

The ETDRS grading scheme is the gold-standard for DR staging. ${ }^{4}$ However, it is labour intensive with low throughput and, thus, has limited applicability in daily practice.

Optical coherence tomography angiography (OCTA) has gained a critical position in the study of DR. ${ }^{5}$ It is a functional extension of structural
OCT, that uses repeated B-scans to detect motion contrast, allowing a dye-less visualisation of retinal microvasculature. ${ }^{6}$ Furthermore, it provides noninvasive three-dimensional mapping of the retinal microvasculature, allowing the identification and quantification of retinal capillary closure, which is the feature that has been shown to better correlate with the clinical features of non-proliferative diabetic retinopathy (NPDR) in its main stages and progression.

There are also indications that information on regional distribution of retinal capillary changes may be particularly relevant in the more advanced stages of the retinopathy. ${ }^{8}$ Studies with widefield fluorescein angiography have shown that midperipheral and peripheral changes in retinal progression need to be considered and may be valuable for determining retinopathy progression. The recent development of commercially available sweptsource OCT (SS-OCT) instruments enables OCTA visualisation of retinal vasculature over larger fields of view, $12 \times 12 \mathrm{~mm}$ or $15 \times 9 \mathrm{~mm} .{ }^{9}$ Also, SS-OCTimaging provides advantages in the speed of acquisition and better penetration, with enhanced resolution and improved sensitivity due to the density of A-scans, compared with conventional spectral domain OCT (SD-OCT) imaging. ${ }^{10}$

This study applies these recent technological advances to examine the value of commercially available SS-OCTA in the characterisation of retinal capillary non-perfusion in NPDR and the contribution of macular and widefield imaging to improve characterisation of the different ETDRS stages of NPDR.

\section{METHODS}

In this prospective cross-sectional study, 105 individuals with diabetes with or without retinopathy and 38 individuals without diabetes were recruited from Association for Innovation and Biomedical Research on Light and Image screening programmes and underwent OCTA imaging and a full ophthalmological examination.

The study exclusion criteria comprised the presence of age-related macular degeneration, glaucoma, vitreomacular disease, high ametropia (spherical equivalent greater than -6 and +2 D), any previous laser treatment or intravitreal injections or any patient comorbidity likely to affect the eye and not related with diabetes or cardiovascular disease. Excluded were also people with type 2 diabetes (T2D) with uncontrolled systemic 
hypertension (values outside normal range: systolic 70-120 mm $\mathrm{Hg}$ and diastolic 50-120 mm Hg), haemoglobin A1c levels above $10 \%$ and history of ischaemic heart disease.

\section{OCTA imaging}

Study subjects were imaged by the SS-OCT PlexElite 9000 (ZEISS, Dublin, California, USA). The acquisition protocols used were the 'Angio $15 \mathrm{~mm} \times 9 \mathrm{~mm}$ ' $(834 \times 500 \times 1536$ voxels with $2 \mathrm{~B}$-Scan repetitions) and the 'Angio $3 \mathrm{~mm} \times 3 \mathrm{~mm}$ ' $(300 \times 300 \times 1536$ voxels with $4 \mathrm{~B}$-scan repetitions) protocols.

Acquired data from the PlexElite was processed by the 'Density Quantification v0.3.5' algorithm available on the Advanced Retina Imaging (ARI) portal which uses multilayer segmentation and calculates vascular density metrics for the superficial capillary plexus (SCP), deep capillary plexus (DCP) and full retina (FR). For retinal mid-periphery areas, to take advantage of the wide-field capabilities of the $15 \times 9 \mathrm{~mm}$ protocol, 3 extra concentric rings were added to the standard 9 ETDRS areas with $31^{\circ}, 42^{\circ}$ and $52^{\circ}$ of field of view (extended 1, extended 2 and extended 3).

Vessel density (VD) is calculated by applying a threshold algorithm to the SCP, DCP and FR angiography en-face images, resulting in a binary image where each pixel corresponds to a perfused or non-perfused area, 0 or 1 , respectively. These images are then skeletonized to represent vessels by their centrelines (traces with 1-pixel width). VD is the average of the skeletonized image over the region of interest scaled by the distance between pixels.

The VD algorithm is expected to perform more poorly when the pixel sampling is lower as is the case for larger fields of view. For this reason, we do not expect the results from the sweptsource and spectral domain $3 \times 3$ scans to give identical results (different wavelengths, bandwidths, and sampling densities), ${ }^{7}$ and we do not expect the density measured in the inner retinal area of the $15 \times 9$ scans to match what is measured in the more densely sampled $3 \times 3$ area. Data from the most densely sampled scan should always be expected to be closer to ground truth, but in the absence of adaptive optics we do not expect any commercial device to give a truly accurate estimate of the overall microvascular density. However, VD has been found to correlate with stage of disease even for less densely sampled scans, so we expect the measurements to be useful in a relative manner-data can be compared across populations and longitudinally, but should always be matched for the same scan and instrument type. Results obtained with SD-OCTA (AngioPlex) and SS-OCTA (PlexElite) for the same eyes and area showed a Pearson correlation of 0.7 (online supplemental table 1).

All OCTA examinations underwent a quality check to discard acquisitions having a signal strength lower than 7 , motion artefacts or evidence of defocus or blur in more than $25 \%$ of the area under analysis.

\section{Retinal thickness and layer segmentation}

The structural data from the same SS-OCTA scanning protocols was also analysed by the 'ETDRS Retinal Thickness v0.1' algorithm available on the ARI portal to calculate total retinal thickness values for each ETDRS standard areas. The average thickness values for the ganglion cell layer +inner plexiform layer (GCL+IPL) were calculated with 'Multilayer Segmentation v0.3' algorithm also available on the ARI portal.

\section{Colour fundus photography}

Colour fundus photography was performed according to the ETDRS protocol using a Topcon TRC 50DX camera (Topcon
Medical Systems, Tokyo, Japan). The DR severity score was determined by two independent graders in a context of an experienced reading centre (Coimbra Ophthalmology Reading Centre, Coimbra, Portugal) using a modification of the Airlie House classification scheme according to the ETDRS Protocol. ${ }^{4}$ This severity scale comprises eight severity levels of the retinopathy.

\section{Statistical analysis}

Statistical analysis was performed using Stata V.12.1 (Stata Corps. LP). Variables were summarised for healthy controls and for each ETDRS group, 10, 20, 35, 43 and 47-53 using mean and SD. The Mann-Whitney U-test was performed to compare the statistically significant differences between VD metrics and retinal thickness for each of the defined groups. Bonferroni correction was applied to VD comparisons setting the statistical significance to $\mathrm{p}<0.0167$, otherwise $\mathrm{p}<0.05$.

\section{RESULTS}

One hundred and five patients with diabetes, one eye per patient, either without retinopathy or with different degrees of retinopathy, were prospectively imaged for this study, using two acquisition protocols, $3 \times 3 \mathrm{~mm}$ and $15 \times 9 \mathrm{~mm}$ from the PlexElite.

Of the 105 eyes of patients with diabetes, 16 eyes had no clinical evidence of retinopathy, 18 eyes were classified as ETDRS grade 20, 39 eyes were identified as grade 35, 17 eyes as grade 43 and 15 eyes were identified as grades 47-53. Thirty-eight healthy subjects were evaluated as control age-matched population. A diagram of the retinal locations examined, and representation of the areas sampled is depicted in figure 1.

Demographics of the population considering the distinct ETDRS levels and OCTA VD metrics are summarised in table 1. The OCTA VD metrics are presented as the mean values $( \pm \mathrm{SD})$ at inner ring quantified by PlexElite, using $3 \times 3 \mathrm{~mm}$ acquisition protocol. The VD metrics in the SCP, DCP and FR identify decrease in VD in all retinal capillary layers in individuals with diabetes. In the macular area, the best correlation between ETDRS grade of retinopathy and VD changes is obtained with the inner ring metrics, representing the parafoveal retinal capillary microvasculature. No significant differences were observed in the total retinal thickness, both at central and inner ring areas, between the different ETDRS grades.

Retinal midperiphery analysis of VD performed using the $15 \times 9 \mathrm{~mm}$ protocol with the SS-OCTA PlexElite showed relevant information identifying involvement of more peripheral regions of the retina in the more advanced ETDRS stages of NPDR. Measurements with the $15 \times 9 \mathrm{~mm}$ protocol were particularly discriminatory as the retinopathy progressed to the 43-53 stages. Particularly, in the extended regions 2 and 3, the ETDRS 43 and 47-53 groups presented average VD values that are lower that ETDRS 20-35 groups (table 2). The thickness of GCL+IPL remained with normal range in the different retina regions examined along the different ETDRS severity stages of NPDR.

Combining the results of the protocols $3 \times 3 \mathrm{~mm}$ and $15 \times 9 \mathrm{~mm}$, it was possible to distinguish two major groups of NPDR representing two major stages of NPDR progression: group A including eyes with diabetes and either no ophthalmological signs of retinopathy (ETDRS 10) and eyes with mild retinopathy (ETDRS 20-35), and group B, including eyes with ETDRS grades $43-53$, showing increased retinal capillary closure in the retina midperiphery (table 3 ). The increase in capillary closure of the more peripheral regions of the retina in the more advanced ETDRS stages involves predominantly the DCP. 
A

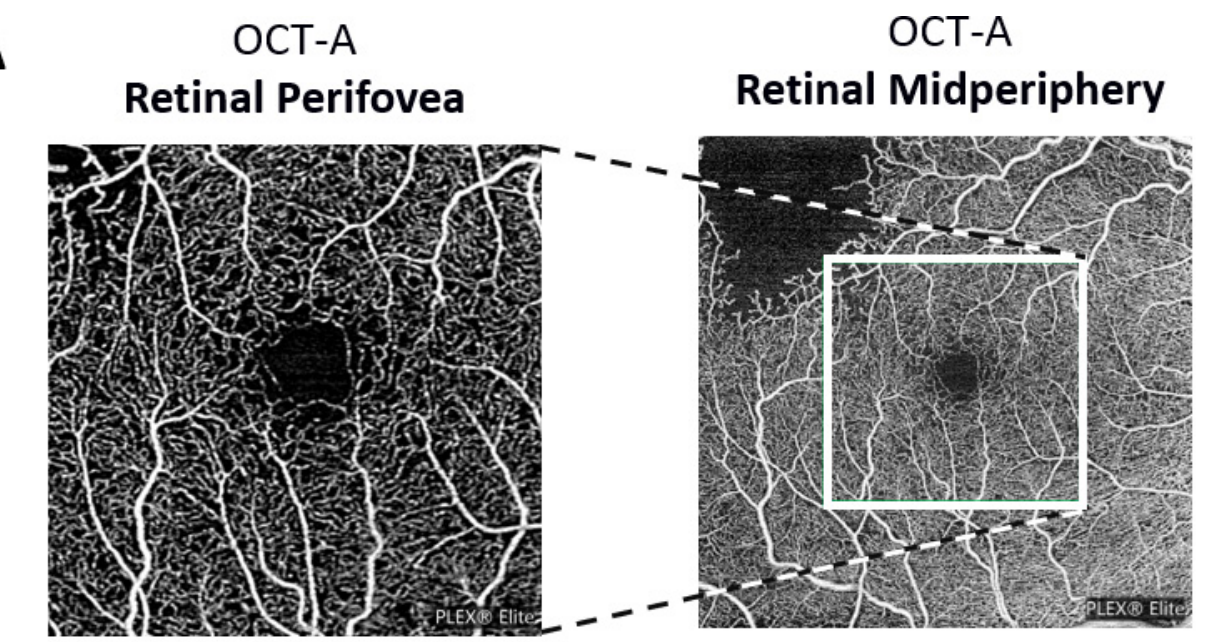

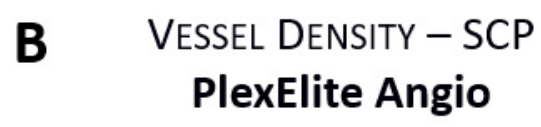

$3 \times 3 \mathrm{~mm}$

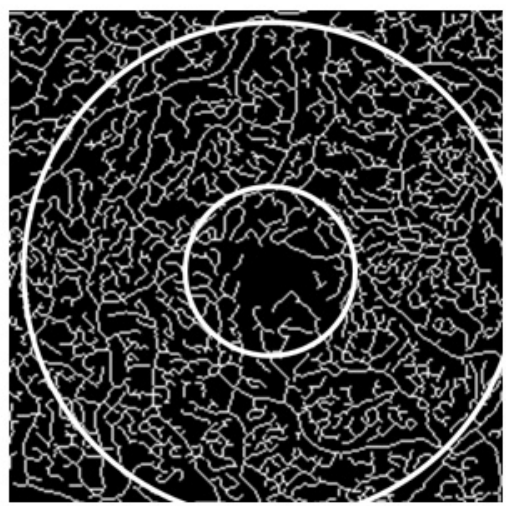

\section{VESSEL DENSITY - SCP \\ PlexElite Angio \\ $15 \times 9 \mathrm{~mm}$}

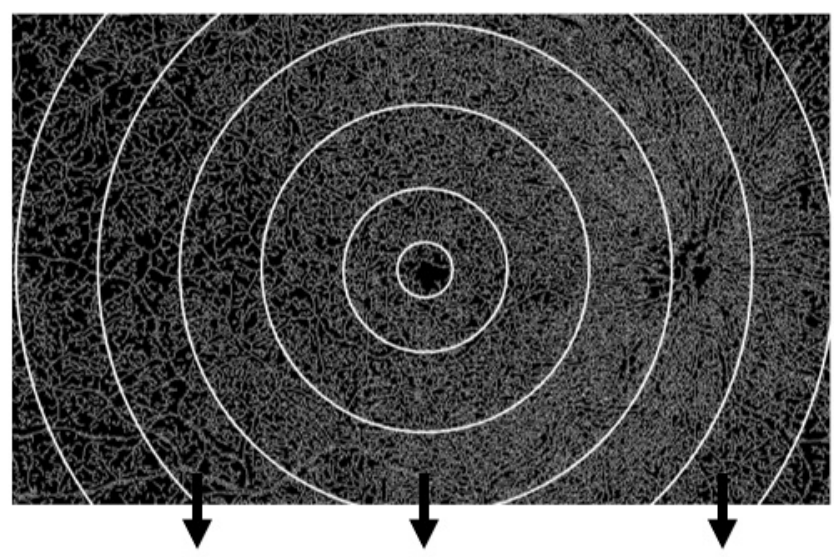

\section{Extended 2 \\ Extended 1 \\ Extended 3}

Figure 1 Representative diagram depicting retinal locations examined with optical coherence tomography angiography (OCTA; perifovea vs midperiphery) (A) and the sample areas acquired with PlexElite Angio $3 \times 3 \mathrm{~mm}$ and $15 \times 9 \mathrm{~mm}$ (B). SCP, superficial capillary plexus.

\section{DISCUSSION}

In previous reports, we have demonstrated that SD-OCTA is able to identify and quantify retinal capillary closure in the initial stages of diabetic retinal disease. ${ }^{711} 12$ These studies were focused on the initial stages of DR identifying changes in the parafoveal retinal circulation. Now, in this study, retinal capillary closure was analysed in diabetic eyes of different disease severity grades using also widefield $(15 \times 9 \mathrm{~mm})$ SS-OCTA images. Our results show that there is a statistically significant increase in the retinal capillary closure in more peripheral regions of the retina as DR severity increases, particularly in ETDRS grades 43-53, an observation that confirms the findings of a recent study using $12 \times 12 \mathrm{~mm}$ SS-OCTA images. ${ }^{9}$ Retinal capillary closure is measured with OCTA skeletonised VD and interpreted as representing individual capillaries that are either absent or have red cell blood flow below the threshold of detection.

Retinal capillary closure was found to be increased in the eyes of persons with T2D evaluated in this study, even in eyes with no retinal changes detected by ophthalmoscopy, that is, ETDRS grade 10 , confirming previous reports. ${ }^{13}$ In eyes with DR, retinal capillary closure increases in parallel with retinopathy severity, ${ }^{12}$ and this progressive increase is better identified in the perifoveal region of the macula in the initial stages of the retinopathy. Involvement of the retinal midperiphery becomes dominant only in more advanced stages of the retinopathy, ETDRS grades 43-53. Fluorescein angiography was not performed in this study because it has been shown in previous work by our group than is clearly less effective in determining capillary closure that OCTA. ${ }^{14}$

Relevant and valuable information about the presence of retinal capillary closure in NPDR is, therefore, obtained by collecting data with the SS-OCTA, which allows data sampling using both protocols, $3 \times 3 \mathrm{~mm}$ and $15 \times 9 \mathrm{~mm}$, and thus obtaining information from the parafoveal region and retinal midperiphery.

Our study suggests that capillary closure occurs very early in diabetic retinal disease and is initiated in the macula. It is only later on, as the disease progresses with remodelling of the retinal circulation and an altered retinal blood flow distribution probably through preferential arteriovenous preferential channels 
Table 1 Baseline demographic characteristics and Angio $3 \times 3 \mathrm{~mm}$ vessel density metrics considering distinct DRSS stages of the disease

\begin{tabular}{|c|c|c|c|c|c|c|c|}
\hline \multicolumn{2}{|l|}{ Demographics } & Healthy & ETDRS 10 & ETDRS 20 & ETDRS 35 & ETDRS 43 & $\begin{array}{l}\text { ETDRS } \\
47-53\end{array}$ \\
\hline \multicolumn{2}{|l|}{ N } & 38 & 16 & 18 & 39 & 17 & 15 \\
\hline \multicolumn{2}{|l|}{ Age (years) } & $65.8 \pm 10.2$ & $68.8 \pm 4.8$ & $64.8 \pm 7.0$ & $66.9 \pm 9.5$ & $66.6 \pm 7.9$ & $65.4 \pm 7.5$ \\
\hline \multicolumn{2}{|c|}{ Sex (Male/female) } & $20 / 18$ & $9 / 7$ & $10 / 8$ & $28 / 11$ & $13 / 4$ & $10 / 5$ \\
\hline \multicolumn{2}{|c|}{ Diabetes duration (years) } & - & $18.5 \pm 11.3$ & $20.8 \pm 13.8$ & $21.6 \pm 10.7$ & $21.7 \pm 5.9$ & $16.2 \pm 8.8$ \\
\hline \multicolumn{2}{|c|}{$\operatorname{HbA1c}(\%)$} & $6.2 \pm 1.0$ & $7.1 \pm 1.1$ & $7.4 \pm 1.3$ & $77.6 \pm 0.8$ & $7.5 \pm 1.3$ & $8.1 \pm 1.1$ \\
\hline \multicolumn{2}{|l|}{ BCVA (letters) } & $83.0 \pm 4.3$ & $83.4 \pm 4.4$ & $84.3 \pm 6.3$ & $84.2 \pm 4.5$ & $82.4 \pm 6.8$ & $80.1 \pm 8.0$ \\
\hline \multicolumn{2}{|l|}{$\mathrm{SCPm} \mathrm{mm} \mathrm{m}^{-1}$} & $14.7 \pm 1.3$ & $\begin{array}{l}12.6 \pm 2.5 \\
p=0.009\end{array}$ & $\begin{array}{l}13.2 \pm 1.7 \\
p=0.005\end{array}$ & $\begin{array}{l}12.1 \pm 2.0 \\
p=0.000\end{array}$ & $\begin{array}{l}12.2 \pm 1.9 \\
p=0.010\end{array}$ & $\begin{array}{l}12.0 \pm 1.3 \\
p=0.001\end{array}$ \\
\hline $\begin{array}{l}\text { Vessel density } \\
\text { DCP, mm-1 }\end{array}$ & $\left(\right.$ Inner ring $\left.(5.10)^{\circ}\right)$ & $14.5 \pm 1.5$ & $\begin{array}{l}11.9 \pm 2.8 \\
p=0.003\end{array}$ & $\begin{array}{l}13.0 \pm 1.6 \\
p=0.012\end{array}$ & $\begin{array}{l}12.9 \pm 2.3 \\
p=0.014\end{array}$ & $\begin{array}{l}12.3 \pm 2.5 \\
p=0.045\end{array}$ & $\begin{array}{l}11.6 \pm 1.2 \\
p=0.001\end{array}$ \\
\hline $\begin{array}{l}\text { Vessel density } \\
\mathrm{FR}, \mathrm{mm}^{-1}\end{array}$ & $\left(\right.$ Inner ring $\left.(5.10)^{\circ}\right)$ & $17.9 \pm 1.1$ & $\begin{array}{l}15.7 \pm 2.8 \\
p=0.009\end{array}$ & $\begin{array}{l}16.6 \pm 1.3 \\
p=0.008\end{array}$ & $\begin{array}{l}16.0 \pm 2.0 \\
p=0.000\end{array}$ & $\begin{array}{l}15.4 \pm 2.0 \\
p=0.010\end{array}$ & $\begin{array}{l}15.2 \pm 1.4 \\
p=0.000\end{array}$ \\
\hline \multirow[t]{2}{*}{$\begin{array}{l}\text { Thickness } \\
\text { retina, } \mu \mathrm{m}\end{array}$} & (Central SF $(0.5)^{\circ}$ ) & $271.4 \pm 20.6$ & $\begin{array}{l}276.3 \pm 29.6 \\
p=0.675\end{array}$ & $\begin{array}{l}277.4 \pm 38.3 \\
p=0.952\end{array}$ & $\begin{array}{l}282.2 \pm 29.0 \\
p=0.201\end{array}$ & $\begin{array}{l}274.8 \pm 23.4 \\
p=0.677\end{array}$ & $\begin{array}{l}305.0 \pm 49.6 \\
p=0-132\end{array}$ \\
\hline & (Inner ring $\left.(5.10)^{\circ}\right)$ & $332.8 \pm 17.7$ & $\begin{array}{l}330.1 \pm 12.7 \\
p=0.646\end{array}$ & $\begin{array}{l}331.7 \pm 14.5 \\
p=0.971\end{array}$ & $\begin{array}{l}328.1 \pm 17.2 \\
p=0.509\end{array}$ & $\begin{array}{l}333.4 \pm 17.8 \\
p=0.850\end{array}$ & $\begin{array}{l}346.3 \pm 30.3 \\
p=0.338\end{array}$ \\
\hline
\end{tabular}

Vessel density values, obtained using PlexElite $3 \mathrm{~mm} \times 3 \mathrm{~mm}$, and retinal thickeness values were calculated using Zeiss MultiLayer segmentation algorithm. Comparisons between diabetic individuals and healthy controls were performed using Mann-Whitney U-test. Bonferroni correction was applied to vessel density comparisons setting the statistical significance to $p<0.0167$, otherwise $p<0.05$.

Data are presented as mean \pm SD.

Statistical significance highlighted in bold.

BCVA, best corrected visual acuity; DCP, deep capillary plexus; FR, full retina; HbA1c, glycated haemoglobin; SCP, superficial capillary plexus.

that capillary closure develops in more peripheral regions of the retina. $^{15}$

Our study shows that the use of SS-OCTA and a combined protocol, $3 \times 3 \mathrm{~mm}$ and $15 \times 9 \mathrm{~mm}$ allows discrimination between different ETDRS grades, identifying two main stages of NPDR, mild versus severe.

The ETDRS classification is based on the presence of different features, as microaneurysms, hard and soft exudates, venous loops, and so on, identified by fundus photography. These alterations are considered to be related to the presence of capillary closure and ischaemia. ${ }^{16}$ It is, therefore, reasonable to think that capillary closure, if reliably quantified and measured, may be an appropriate indicator of changes identified by fundus photography and become an alternative to the ETDRS classification.

Considering that only two-step changes in ETDRS severity grading have been shown to be clinically significant, we propose that ETDRS groups assembled using in group A, grades 10-35 and in group B, grades 43-53, corresponding to no and mild retinopathy in group A and moderate to severe in group B, is a promising approach and may represent objectively and more accurately the real progression of NPDR. The use of SS-OCTA, with combined protocols, capable of detecting early capillary closure in the perifoveal region and later capillary closure in the midperiphery, may offer, therefore, a simple to use alternative to the laborious and demanding ETDRS grading process which classifies a complex set of parameters. The results here reported give, indeed, a practical basis for the International Classification of Diabetic Retinopathy. ${ }^{17}$ Hence, we propose a numerical threshold to separate mild NPDR from moderate and severe NPDR. Skeletonised VD in the SCP (inner ring protocol, $3 \times 3 \mathrm{~mm}$ ) lower than 13 (mean of healthy controls less $1 \mathrm{SD}$ ) and higher than 10 (mean of healthy controls less 1 SD) in the DCP midperiphery (protocol $15 \times 9$, extended area 3 ) identifies mild NPDR. On the other hand, skeletonised VD lower than 13 in the SCP (inner ring protocol, $3 \times 3 \mathrm{~mm}$ ) and lower than 10 in the DCP midperiphery (protocol $15 \times 9$, extended area 3 ) identifies moderate and severe NPDR (respectively AUC: 0.83;
0.63; online supplemental figure 1 ). This identification is particularly relevant for clinical practice as mild NPDR has a 5-year incidence of vision-threatening complications of approximately $10 \%{ }^{18}$ much lower than the 5 -year incidence of moderate and severe NPDR. ${ }^{19}$

This study confirms that regional distribution of the DR lesions in the retina may reflect risk factors and may be important in defining the stage of DR. ${ }^{20} 21$ Capillary closure in the midperiphery in a diabetic retina is indicative of an advanced stage of retinopathy, whereas capillary closure limited to the perifovea suggests a milder stage of the disease. Furthermore, regional distribution of DR lesions has led Hove $e t a l^{8}$ to suggest that future improvements to existing grading systems should focus on the quantification of overall retinopathy lesions in the early stages and the regional distribution of retinopathy lesions in the more advanced stages retinopathy. Red blood cell flow in retina and brain capillaries has been shown to be redistributed between thoroughfare channels, capillaries with high resting velocity and exchange capillaries, capillaries characterised by low resting velocity. $^{22}$ Pre-existing preferential arteriovenous connections may act as shunts that bypass the occluded retinal microcirculation. $^{23-25}$ These concepts are in line with our findings, again emphasising the potential of OCTA nonperfusion metrics to identify DR progression.

The location of retinal capillary closure in the different retinal capillary layers in the initial stages of DR has been a matter of controversy. ${ }^{71}$ Some authors have found evidence for initial retinal non-perfusion changes in the SCP, whereas others found that the DCP is the first retinal capillary plexus to show nonperfusion. ${ }^{26}$ The division and separation of the retinal capillary network in two, three or four layers have also been a matter of controversy. ${ }^{27} 28$ In this study, retinal capillary closure predominates initially in the SCP with progressive later involvement of the DCP. The involvement of the DCP becomes predominant as the DR progresses in severity. This finding may have important implications to our understanding of the pathophysiology of DR. It must be taken into account, however, that the 


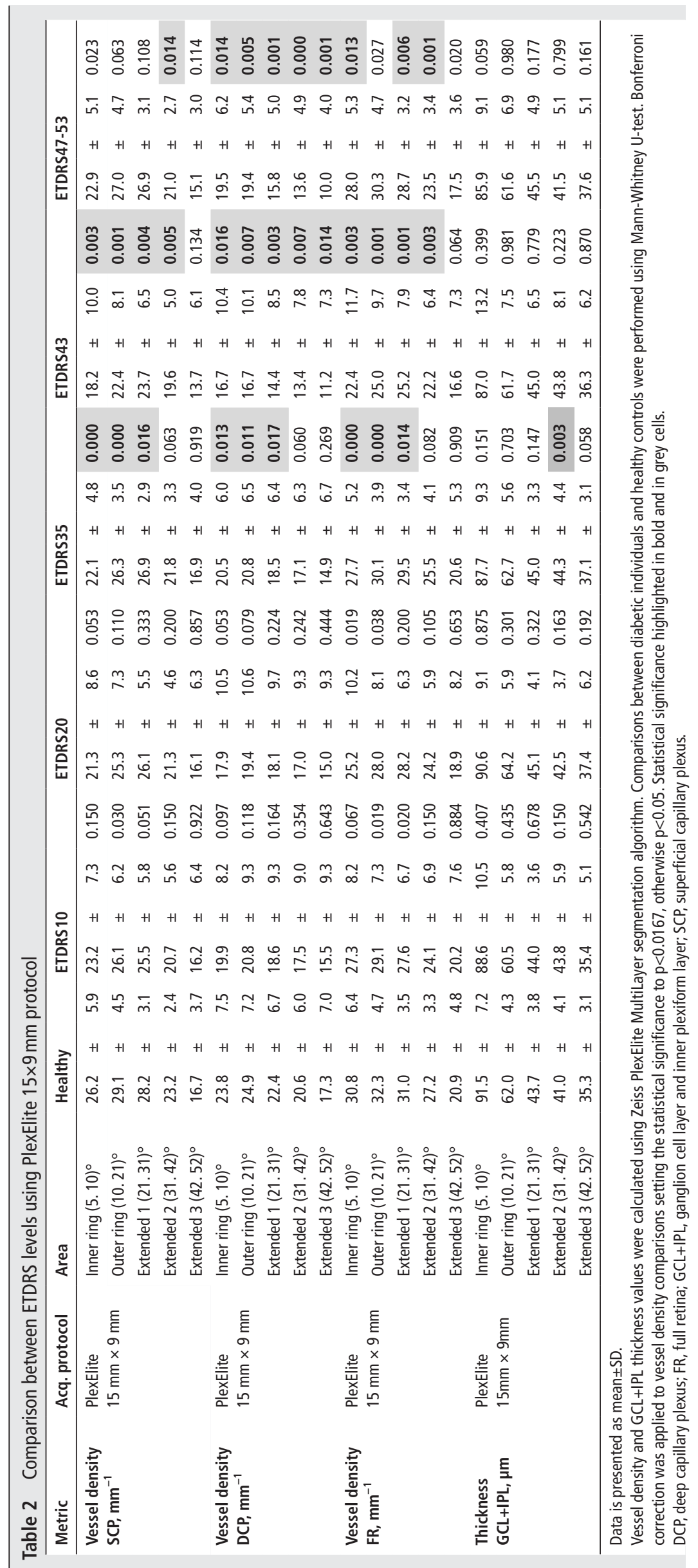


Table 3 Comparison between ETDRS groups assembled by severity level

\begin{tabular}{|c|c|c|c|c|c|c|c|}
\hline Metric & Acq. protocol & Area & Healthy & $\begin{array}{l}\text { Healthy vs } \\
\text { ETDRS10-35 }\end{array}$ & ETDRS10-35 & $\begin{array}{l}\text { ETDRS } 10-35 \text { vs } \\
\text { ETDRS43-53 }\end{array}$ & ETDRS43-53 \\
\hline \multirow{4}{*}{$\begin{array}{l}\text { Vessel density } \\
\text { SCP, } \mathrm{mm}^{-1}\end{array}$} & PlexElite $3 \mathrm{~mm} \times 3 \mathrm{~mm}$ & Inner ring $(5,10)^{\circ}$ & $14.7 \pm 1.3$ & 0.000 & $12.6 \pm 1.9$ & 0.017 & $11.9 \pm 1.5$ \\
\hline & \multirow[t]{3}{*}{ PlexElite $15 \mathrm{~mm} \times 9 \mathrm{~mm}$} & Extended $1(21,31)^{\circ}$ & $28.2 \pm 3.1$ & 0.018 & $26.4 \pm 4.3$ & 0.304 & $24.9 \pm 5.5$ \\
\hline & & Extended $2(31,42)^{\circ}$ & $23.2 \pm 2.4$ & 0.047 & $21.5 \pm 4.2$ & 0.137 & $20.2 \pm 4.3$ \\
\hline & & Extended $3(42,52)^{\circ}$ & $16.7 \pm 3.7$ & 0.976 & $16.5 \pm 5.2$ & 0.059 & $14.3 \pm 5.1$ \\
\hline \multirow{4}{*}{$\begin{array}{l}\text { Vessel density } \\
\text { DCP, } \mathrm{mm}^{-1}\end{array}$} & PlexElite $3 \mathrm{~mm} \times 3 \mathrm{~mm}$ & Inner ring $(5,10)^{\circ}$ & $14.5 \pm 1.5$ & 0.000 & $12.7 \pm 2.2$ & 0.003 & $11.6 \pm 1.6$ \\
\hline & \multirow[t]{3}{*}{ PlexElite $15 \mathrm{~mm} \times 9 \mathrm{~mm}$} & Extended $1(21,31)^{\circ}$ & $22.4 \pm 6.7$ & 0.023 & $18.4 \pm 7.9$ & 0.046 & $15.1 \pm 7.4$ \\
\hline & & Extended $2(31,42)^{\circ}$ & $20.6 \pm 6.0$ & 0.071 & $17.2 \pm 7.6$ & 0.032 & $13.6 \pm 6.8$ \\
\hline & & Extended $3(42,52)^{\circ}$ & $17.3 \pm 7.0$ & 0.288 & $15.1 \pm 7.9$ & 0.015 & $10.9 \pm 6.2$ \\
\hline \multirow{4}{*}{$\begin{array}{l}\text { Vessel density } \\
\mathrm{FR}, \mathrm{mm}^{-1}\end{array}$} & PlexElite $3 \mathrm{~mm} \times 3 \mathrm{~mm}$ & Inner ring $(5,10)^{\circ}$ & $17.9 \pm 1.1$ & 0.000 & $16.1 \pm 2.0$ & 0.002 & $15.2 \pm 1.4$ \\
\hline & \multirow[t]{3}{*}{ PlexElite $15 \mathrm{~mm} \times 9 \mathrm{~mm}$} & Extended $1(21,31)^{\circ}$ & $31.0 \pm 3.5$ & 0.009 & $28.8 \pm 5.0$ & 0.062 & $26.5 \pm 6.6$ \\
\hline & & Extended $2(31,42)^{\circ}$ & $27.2 \pm 3.3$ & 0.042 & $24.8 \pm 5.3$ & 0.030 & $22.7 \pm 5.4$ \\
\hline & & Extended $3(42,52)^{\circ}$ & $20.9 \pm 4.8$ & 0.861 & $20.1 \pm 6.6$ & 0.016 & $17.0 \pm 6.1$ \\
\hline \multirow{4}{*}{$\begin{array}{l}\text { Thickness } \\
\text { GCL+IPL, } \mu \mathrm{m}\end{array}$} & PlexElite $3 \mathrm{~mm} \times 3 \mathrm{~mm}$ & Inner ring $(5,10)^{\circ}$ & $93.7 \pm 8.2$ & 0.324 & $88.2 \pm 11.6$ & 0.747 & $84.0 \pm 21.4$ \\
\hline & \multirow[t]{3}{*}{ PlexElite $15 \mathrm{~mm} \times 9 \mathrm{~mm}$} & Extended $1(21,31)^{\circ}$ & $43.7 \pm 3.8$ & 0.184 & $44.8 \pm 3.6$ & 0.897 & $45.3 \pm 5.9$ \\
\hline & & Extended $2(31,42)^{\circ}$ & $41.0 \pm 4.1$ & 0.007 & $43.7 \pm 4.6$ & 0.188 & $43.1 \pm 7.1$ \\
\hline & & Extended $3(42,52)^{\circ}$ & $35.3 \pm 3.1$ & 0.167 & $36.8 \pm 4.5$ & 0.787 & $37.0 \pm 5.9$ \\
\hline
\end{tabular}

Data are presented as mean \pm SD.

Vessel density and GCL+IPL thickness values were calculated using Zeiss PlexElite MultiLayer segmentation algorithm. Comparisons between individuals with diabetes and healthy controls were performed using Mann-Whitney U-test. Bonferroni correction was applied to vessel density comparisons setting the statistical significance to $\mathrm{p}<0.0167$, otherwise $\mathrm{p}<0.05$. Statistical significance highlighted in bold and in grey cells.

DCP, deep capillary plexus; FR, full retina; GCL+IPL, ganglion cell layer and inner plexiform layer; SCP, superficial capillary plexus.

actual segmentation of the retina in different OCTA equipment is different which may explain the different findings of different research groups. It may be that for clinical purposes, the best option is to quantify in a way that is independent of the segmentation, that is, using the full retina instead of some definition of the superficial or deeper layers.

The GCL+IPL shows similar degrees of thinning that is, neurodegeneration, in eyes with different degrees of nonperfusion. It is of particular interest to register that the increase in nonperfusion present in the more peripheral regions of the retina, in more severe stages of the retinopathy occurs without increased thinning of the GCL+IPL demonstrating that the nonperfusion is associated with an ischaemic situation and is not the end result of retinal tissue atrophy.

Our study has several limitations. The sample size is relatively small with relatively few patients having severe NPDR, in contrast with a previous report using widefield images with SS-OCTA. ${ }^{9}$ Also, the $15 \times 9 \mathrm{~mm}$ field of view, while widefield in the context of previously reported OCTA imaging is still a relatively small field compared with widefield fluorescein angiography imaging, and more information may therefore be obtained when more peripheral retina images are collected.

However, our study has also important advantages over previous reports. The distribution of eyes is balanced across the different ETDRS grades. The eyes examined are naïve eyes that had not been previously submitted to any local treatment such as laser or steroid or anti-vascular endotelhial growth factor intravitreal injection. We also examined the different retinal capillary plexus, superficial and deep, as well as the full retina, to account for projection artefacts.

In conclusion, we have performed a study combining regional examination of the retinal circulation, including the macula region and the retinal midperiphery, in patients with T2D in eyes with no retinopathy and different ETDRS grades of NPDR. The study shows that retinal capillary closure is an early finding in the macular area of the diabetic retina and that this alteration increases as the retinopathy progresses in severity involving later more peripheral regions of the retina. Furthermore, SS-OCTA metrics of retinal capillary closure, allowing measurements to be performed in the macula and in more peripheral regions of the retina, may offer an objective and easier to perform alternative to ETDRS severity grading.

Contributors TS, ARS, IPM, LGM and MHM collected and analysed data, reviewed and edited the manuscript. LHW, and SK and LdS analysed data and contributed to writing and editing the manuscript. MD reviewed and edited the manuscript. JGC-V analysed data and wrote the manuscript. JGC-V is the guarantor of this work and, as such, had full access to all the data in the study and takes responsibility for the integrity of the data and the accuracy of the data analysis.

Funding This work was supported by AIBILI and by COMPETE Portugal2020 and by Fundo de Inovação, Tecnologia e Economia Circular (FITEC) - Programa Interface (FITEC/CIT/2018/2).

Competing interests TS, ARS, IPM, LGM and MHM do not have financial disclosures. LHW, SK, LdS and MD: Carl Zeiss Meditec (E). JGC-V reports grants from Carl Zeiss Meditec and is consultant for Alimera Sciences, Allergan, Bayer, Gene Signal, Novartis, Pfizer, Precision Ocular, Roche, Sanofi-Aventis, Vifor Pharma, and Carl Zeiss Meditec.

Patient consent for publication Not required.

Ethics approval The tenets of the Declaration of Helsinki were followed, approval was obtained from the Institutional Ethical Review Board, and written informed consent to participate in the study was obtained from all individuals after the procedures were explained.

Data availability statement Data are available upon reasonable request. The dataset used for this publication was gathered from 105 diabetic patients and 38 healthy control volunteers. The dataset is composed of demographic data (gender, age) and clinical data (diabetes duration, glycated haemoglobin, best corrected visual acuity, layer retinal thickness and vessel density). Data are available upon reasonable request to José Cunha-Vaz, cunha-vaz@aibili.pt, https://orcid.org/00000002-0947-9850

Supplemental material This content has been supplied by the author(s). It has not been vetted by BMJ Publishing Group Limited (BMJ) and may not have been peer-reviewed. Any opinions or recommendations discussed are solely those of the author(s) and are not endorsed by BMJ. BMJ disclaims all liability and responsibility arising from any reliance placed on the content. Where the content includes any translated material, BMJ does not warrant the accuracy and reliability of the translations (including but not limited to local regulations, clinical guidelines, terminology, drug names and drug dosages), and is not responsible for any error and/or omissions arising from translation and adaptation or otherwise 
Open access This is an open access article distributed in accordance with the Creative Commons Attribution Non Commercial (CC BY-NC 4.0) license, which permits others to distribute, remix, adapt, build upon this work non-commercially, and license their derivative works on different terms, provided the original work is properly cited, appropriate credit is given, any changes made indicated, and the use is non-commercial. See: http://creativecommons.org/licenses/by-nc/4.0/.

ORCID iDs

Ana Rita Santos http://orcid.org/0000-0003-3761-3292

Jose G Cunha-Vaz http://orcid.org/0000-0002-0947-9850

\section{REFERENCES}

1 Bourne RRA, Stevens GA, White RA, et al. Causes of vision loss worldwide, 19902010: a systematic analysis. Lancet Glob Health 2013;1:e339-49.

2 Zheng $\mathrm{Y}$, He M, Congdon N. The worldwide epidemic of diabetic retinopathy. Indian J Ophthalmol 2012;60:428-31. doi:10.4103/0301-4738.100542

3 Ferris FL. Diabetic retinopathy. In: Diabetes care, 1993.

4 Grading diabetic retinopathy from stereoscopic color fundus Photographs-An extension of the modified Airlie house classification: ETDRS report number 10. Ophthalmology 1991.

5 Bandello F, Cicinelli MV. 19Th EURETINA Congress Keynote Lecture: diabetic retinopathy today. Ophthalmologica 2020;243:163-71.

6 Spaide RF, Fujimoto JG, Waheed NK, et al. Optical coherence tomography angiography. Prog Retin Eye Res 2018;64:1-55.

7 Marques IP, Alves D, Santos T, et al. Multimodal imaging of the initial stages of diabetic retinopathy: different disease pathways in different patients. Diabetes 2019;68:648-53.

8 Hove MN, Kristensen JK, Lauritzen T, et al. The relationships between risk factors and the distribution of retinopathy lesions in type 2 diabetes. Acta Ophthalmo/ Scand 2006;84:619-23.

9 Alibhai AY, De Pretto LR, Moult EM, et al. Quantification of retinal capillary nonperfusion in diabetics using wide-field optical coherence tomography angiography. Retina 2020;40:412-20.

10 Vira J, Marchese A, Singh RB, et al. Swept-source optical coherence tomography imaging of the retinochoroid and beyond. Expert Rev Med Devices 2020;17:413-26.

11 Durbin MK, An L, Shemonski ND, et al. Quantification of retinal microvascular density in optical coherence tomographic angiography images in diabetic retinopathy. JAMA Ophthalmol 2017;135:370.

12 Marques IP, Alves D, Santos T, et al. Characterization of disease progression in the initial stages of retinopathy in type 2 diabetes: a 2-year longitudinal study. Invest Ophthalmol Vis Sci 2020;61:20.
13 Choi W, Waheed NK, Moult EM, et al. Ultrahigh speed swept source optical coherence tomography angiography of retinal and choriocapillaris alterations in diabetic patients with and without retinopathy. Retina 2017;37:11-21.

14 Soares M, Neves C, Marques IP, et al. Comparison of diabetic retinopathy classification using fluorescein angiography and optical coherence tomography angiography. Br J Ophthalmol 2017;101:62-8.

15 Cunha-Vaz JG. Pathophysiology of diabetic retinopathy. Br J Ophthalmol 1978;62:351-5. doi:10.1136/bjo.62.6.351

16 Kowluru RA, Chan P-S. Capillary dropout in diabetic retinopathy. In: Diabetic retinopathy. Humana Press, 2008: 265-82.

17 Wilkinson CP, Ferris FL, Klein RE, et al. Proposed International clinical diabetic retinopathy and diabetic macular edema disease severity scales. Ophthalmology 2003;110:1677-82. doi:10.1016/S0161-6420(03)00475-5

18 Marques IP, Madeira MH, Messias AL, et al. Retinopathy phenotypes in type 2 diabetes with different risks for macular edema and proliferative retinopathy. J Clin Med 2020;9:1433.

19 Willis JR, Doan QV, Gleeson M, et al. Vision-related functional burden of diabetic retinopathy across severity levels in the United States. JAMA Ophthalmol 2017;135:926.

20 Dobree JH. Simple diabetic retinopathy. evolution of the lesions and therapeutic considerations. Br J Ophthalmol 1970;54:1-10.

21 Bek T, Helgesen A. The regional distribution of diabetic retinopathy lesions may reflect risk factors for progression of the disease. Acta Ophthalmol Scand 2001;79:501-5.

22 Fagrell B, Jörneskog G, Intaglietta M. Disturbed microvascular reactivity and shunting a major cause for diabetic complications. Vasc Med 1999;4:125-7.

23 Cogan DG, Kuwabara T. Capillary shunts in the pathogenesis of diabetic retinopathy. Diabetes 1963;12:293-300.

24 Keith CG, Cunha-Vaz JG, Shakib M. Studies on the effects of osmotically active substances on the circulation and structure of the retina. I. Observations in vivo. Invest Ophthalmol 1967;6:1.

25 Hudetz AG, Fehér G, Weigle CG, et al. Video microscopy of cerebrocortical capillary flow: response to hypotension and intracranial hypertension. Am J Physiol 1995;268:H2202-10.

26 Rosen RB, Andrade Romo JS, Krawitz BD, et al. Earliest evidence of preclinical diabetic retinopathy revealed using optical coherence tomography angiography perfused capillary density. Am J Ophthalmol 2019;203:103-15.

27 Park JJ, Soetikno BT, Fawzi AA. Characterization of the middle capillary plexus using optical coherence tomography angiography in healthy and diabetic eyes. Retina 2016;36:2039-50

28 Forte R, Haulani H, Jürgens I. Quantitative and qualitative analysis of the three capillary plexuses and choriocapillaris in patients with type 1 and type 2 diabetes mellitus without clinical signs of diabetic retinopathy: a prospective pilot study. Retina 2020;40:333. 